\title{
Binary Eclipsing Millisecond Pulsars: A Decade of Timing
}

\author{
David J. Nice \\ Physics Department, Princeton University, Box 708, Princeton, NJ \\ $08544 U S A$ \\ Zaven Arzoumanian \\ NASA-Goddard Space Flight Center, Mailstop 662.0, Greenbelt, MD \\ 20771 ISA \\ Stephen E. Thorsett \\ Department of Astronomy and Astrophysics, University of California, \\ Santa Cruz, CA 95064 USA
}

\begin{abstract}
We present results of long-term timing of eclipsing binaries PSR B1744-24A and PSR B1957+20 at Arecibo, the VLA, and Green Bank. Both pulsars exhibit irregularities in pulsar rotation and orbital motion. Increases and decreases of the orbital period of PSR B1957+20 are of order $\Delta P_{b} / P_{b} \sim 10^{-7}$, varying on a time scale of a few years. Over a decade of observations, the orbital period of PSR B1744-24A has only decreased, with time scale $\left|P_{b} / \dot{P}_{b}\right| \sim 200$ Myr. When the effects of orbital motion are removed from the timing data, long-term trends remain in the pulse phase residuals, with amplitudes of order 30 and $500 \mu \mathrm{s}$, respectively, for $\mathrm{B} 1957+20$ and B1744-24A. Such large "timing noise" is not seen in other spun-up pulsars (isolated or binary), leading us to conclude that it is a consequence of mass flow in the system. Possible causes include variations in the rotation of the pulsars and movement of the binary systems along the line of sight (perhaps due to gravitational interactions with outflowing matter).
\end{abstract}

\section{Introduction}

There are now seven known eclipsing binary pulsars (see Table 1). In each system the eclipses are longer than expected for a secondary confined to its Roche lobe. From this it is inferred that there is continuous mass flow from the secondary, constantly replenishing a volume larger than its Roche lobe. The mass loss may be due to Roche lobe overflow, stellar wind, or a combination, and in some cases is induced by heating of the secondary by pulsar irradiation. Observation of ionized material at eclipse edges, detected as dispersion measure increases, confirms this picture.

These systems, or systems similar to them, form a crucial link in the evolution of low-mass neutron star binaries into isolated pulsars. Common characteristics include short orbits ( 2 to 10 hours) and light secondaries $\left(0.02\right.$ to $\left.0.15 \mathrm{M}_{\odot}\right)$. 
Table 1. Eclipsing Binary Pulsars

\begin{tabular}{lrcccc}
\hline \multicolumn{1}{c}{ Pulsar } & $\begin{array}{c}\text { Pulsar } \\
\text { Period } \\
(\mathrm{ms})\end{array}$ & $\begin{array}{c}\text { Orbital } \\
\text { Period } \\
(\text { hr })\end{array}$ & $\begin{array}{c}\mathrm{M}_{2}{ }^{*} \\
\left(\mathrm{M}_{\odot}\right)\end{array}$ & $\begin{array}{c}\text { Globular } \\
\text { Cluster }\end{array}$ & References \\
\hline B1718-19 & 1004.04 & 6.2 & 0.13 & NGC 6342 & 1 \\
B1744-24A & 11.56 & 1.8 & 0.10 & Ter 5 & 2,3 \\
B1957+20 & 1.61 & 9.2 & 0.03 & & 4,5 \\
J 2051-0827 & 4.51 & 2.4 & 0.03 & & 6,7 \\
47 Tuc J & 2.10 & 2.9 & 0.02 & 47 Tuc & 8 \\
47 Tuc O & 2.64 & 3.3 & 0.03 & 47 Tuc & 8 \\
47 Tuc R & 3.48 & 1.5 & 0.03 & 47 Tuc & 8 \\
\hline \hline
\end{tabular}

* Assuming $\mathrm{M}_{1}=1.35 \mathrm{M}_{\odot}$, inclination $60^{\circ}$.

References: 1. Lyne et al. (1993). 2. Lyne et al. (1990).

3. Nice \& Thorsett (1992). 4. Fruchter, Stinebring, \& Taylor (1989).

5. Arzoumanian, Fruchter, \& Taylor (1994) 6. Stappers et al. (1996).

7. Stappers et al. (1998). 8. Camilo et al. (2000).

Mass loss rates estimated from the dispersion and attenuation of radio signals traveling through the ionized wind suggest the secondaries will not be ablated within a Hubble time, unless there is a large, undetected neutral component to the wind. The key question of interest is: how do these systems evolve? Can they, in fact, evaporate their secondaries, or are there similar systems that can? Can planets form from the mass outflow? And how are they related to low mass X-ray binaries, such as SAX J1808.4-3658 (van der Klis, this volume)?

\section{Observations}

We have made timing observations of two eclipsing binaries over a span of ten years. Observations of PSR B1957+20 were made at Arecibo at $430 \mathrm{MHz}$ from 1988 to 1994 and at Green Bank at $575 \mathrm{MHz}$ from 1994 to 1999. Observations of PSR B1744-24A were made at the VLA at $1660 \mathrm{MHz}$ from 1990 to 1999 and at Green Bank at 800,1330, and $1600 \mathrm{MHz}$, also from 1990 to 1999.

We used TEMPo for our initial analysis of pulse arrival times. After removing the standard spin-down and Keplerian orbital model, systematic residual arrival times remained. We analyzed these residuals by fitting small subsets of the data for offsets in pulsar rotation phase, orbital phase, and orbit size (projected semi-major axis). For a uniformly sampled orbit, these phenomena perturb the timing data in orthogonal ways: a pulse rotation offset uniformly affects arrival times at all orbital phases; an orbital phase offset induces excess delays proportional to $\cos \left(\phi_{b}\right)$, where $\phi_{b}$ is the orbital phase, measured from the ascending node; and an orbital size change induces excess delay proportional to $\sin \left(\phi_{b}\right)$. At Green Bank and the VLA, entire orbits were observed on a single day, so we independently analyzed each observing epoch. Arecibo observations had poor orbital coverage on any given day, so we analyzed these data in intervals of 100 days. Results of this analysis are given in Figures 1 and 2. 
(a) Pulse Phase Offset $(\mu s)$

(b) Orbital Phase Offset (sec)

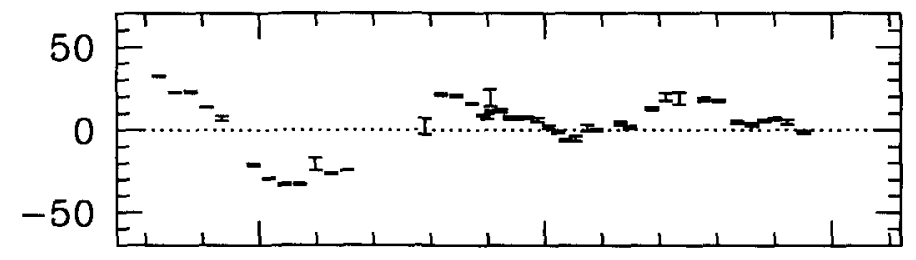

(c) $\begin{aligned} \Delta \mathrm{P}_{\mathrm{b}} / \mathrm{P}_{\mathrm{b}} \\ \left(10^{-7}\right)\end{aligned}$

(c) $\begin{aligned} \Delta \mathrm{P}_{\mathrm{b}} / \mathrm{P}_{\mathrm{b}} \\ \left(10^{-7}\right)\end{aligned}$

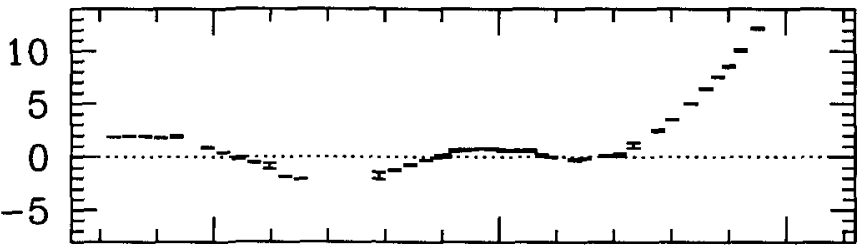

(d) $\Delta x / x$ $\left(10^{-5}\right)$

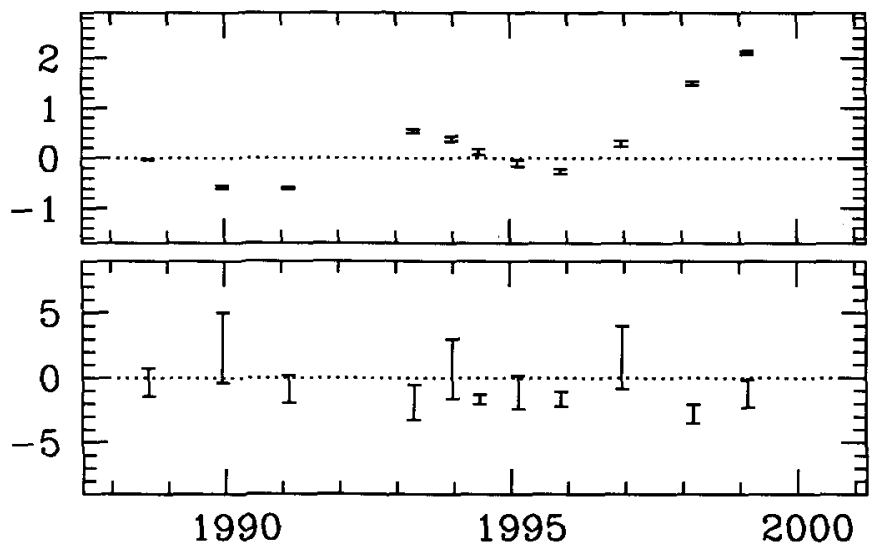

Figure 1. Timing results for PSR B1957+20. Data through 1994 are Arecibo $430 \mathrm{MHz}$, after 1994 are Green Bank $575 \mathrm{MHz}$. (a,b) Pulse phase offset and orbital phase offset at each observing epoch (Green Bank), or averaged over 100 day intervals (Arecibo). (c,d) Orbital period offset (derivative of figure b) and orbital size offset, calculated by averaging sets of four observing epochs (Green Bank) or $\mathbf{4 0 0}$ days (Arecibo). 


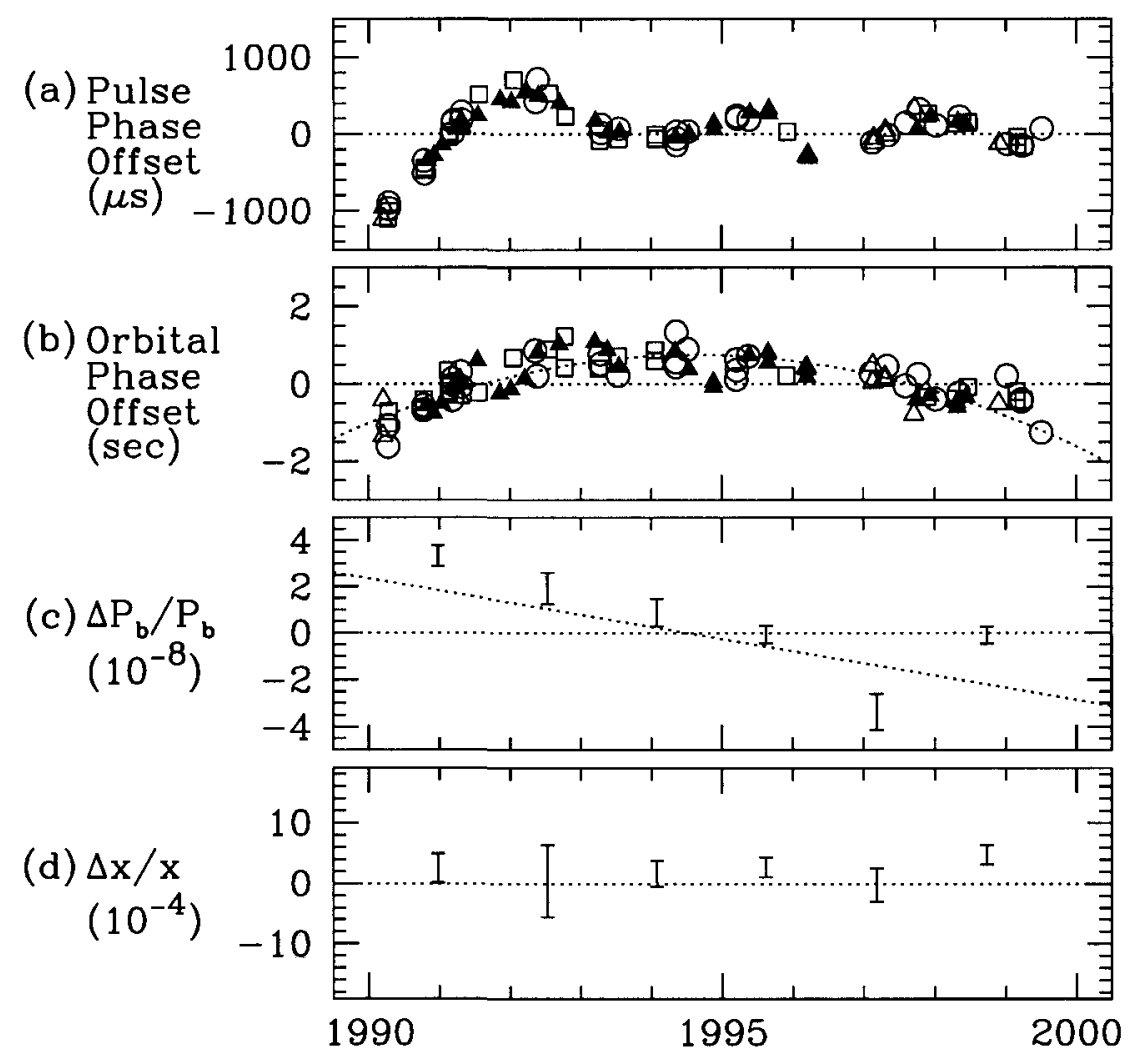

Figure 2. Timing results for PSR B1744-24A. (a,b) Pulse phase offset and orbital phase offset at each observing epoch. Symbols represent Green Bank $800 \mathrm{MHz}$ (circles), Green Bank $1300 \mathrm{MHz}$ (squares) Green Bank $1600 \mathrm{MHz}$ (open triangles), and VLA $1660 \mathrm{MHz}$ (filled triangles). Uncertainties are typically $100 \mu \mathrm{s}$ in pulse phase and $0.6 \mathrm{~s}$ in orbital phase. (c,d) Orbital period offset (derivative of figure b) and orbital size offset, calculated by fitting arrival times within six equal size subsets of data. Dashed lines show best-fit model with constant orbital period derivative. 


\section{Discussion: PSR B1957+20}

The PSR B1957+20 system is undergoing apparently random variations in orbital period, with magnitude $\Delta P_{b} / P_{b} \sim 10^{-7}$ and a time scale of $\sim 5$ years (Figure 1c). This agrees with the conclusion of Arzoumanian, Fruchter, \& Taylor (1994), who analyzed the Arecibo data from 1988 to 1993 . They found the orbital period first decreased and then increased, and predicted that the binary period would exhibit "small quasi-periodic oscillations." A possible source of the period variations is deformation of the secondary by magnetic activity, which in turn is produced by dissipation of tidal energy (Applegate \& Shaham 1994).

Modest "timing noise" is evident in the pulsar signal (Figure 1a). This could be simple variation in dispersion measure-this source has not been regularly observed at multiple frequencies. However, similarity to the PSR B1744-24A timing noise (see below) suggests the origin may lie within the system itself.

\section{Discussion: PSR B1744-24A}

The orbital perturbations of PSR B1744-24A, of order $\Delta P_{b} / P_{b} \sim 10^{-8}$, are smaller than those of PSR B1957+20. A secular downward trend fits the data reasonably well, though not perfectly (Figures $2 b, c$ ). (The specific model plotted was derived from a timing fit to all data, and is essentially equivalent to a quadratic fit to the data in Figure 2b.) The evolutionary time scale inferred from this model is $\left|P_{b} / \dot{P}_{b}\right|_{\text {obs }}=200 \mathrm{Myr}$. This is within an order of magnitude of the orbital decay time scale from gravitational radiation, $\left|P_{b} / \dot{P}_{b}\right|_{\text {GR }}=1000 \mathrm{Myr}$. It seems likely that GR plays a substantial role in the evolution of this system.

The pulsar phase residuals of PSR B1744-24A pose an interesting puzzle. There are systematic variations in pulse arrival time of order $\sim 1 \mathrm{~ms}$ (Figure $2 \mathrm{a}$ ). These excess residuals are largely independent of frequency. (Small frequencydependent differences in arrival times, interpreted as slow changes in dispersion measure, have been removed from the figure.) There are several possible origins of these residuals:

1. "Timing noise" intrinsic to the pulsar rotation. However, timing noise of this magnitude is not seen in other spun-up pulsars. For example, the relatively noisy residuals of PSR B1937+21 reported by Kaspi, Taylor, \& Ryba (1994) show a peak-to-peak excursion of only $7 \mu$ s over more than 8 years, more than two orders of magnitude smaller than the residuals we measure for PSR B1744-24A.

2. Changes in the viewing geometry of the emission region relative to the line of sight. PSR B1744-24A is expected to precess at a rate of $\sim 1.1^{\circ} / \mathrm{yr}$, so a substantial change in viewing angle is possible over 10 years of observations. However, the pulsar has presumably been spun up by accretion of mass and angular momentum from the secondary, aligning the pulsar's angular momentum with the orbital angular momentum. With this near alignment, precession effects would be difficult to detect.

3. Changes in the distance between the entire binary system (pulsar and secondary) and the Earth. This could be caused by a lumpy "excretion disk", 
which could serve as a precursor to planet formation around the pulsar (Banit \& Shaham 1992; Banit et al. 1993). The data are suggestive towards this end - a periodic $1 \mathrm{~ms}$ signal in the residuals, with a period of $5 \mathrm{yr}$, would correspond to an orbit of a $0.3 \mathrm{M}_{\oplus}$ object about the binary system. However, there are no clean periodic signals, so a model like this must invoke a stochastic "background" of lumps (asteroids?).

4. Torques on the pulsar due to infalling matter. The eclipses in this system are highly variable, and the pulsar signal sometimes disappears for several orbits (or possibly longer). This suggests mass may be flowing close to the pulsar, which could be accreted or interact via the "propeller effect", in either case causing a torque on the pulsar, changing the rotation period. Such accretion might be identifiable by X-ray observations of the source at times when the radio signal is not visible. Intriguingly, bursts were seen from this general direction by the Hakucho satellite in 1980 (Makishima et al. 1981), but due to low angular resolution of this telescope and confusion from sources in the dense Galactic center region, any connection between the bursts and PSR B1744-24A must be considered speculative.

Acknowledgments. Much of the $1957+20$ data from Arecibo was collected by A. S. Fruchter, M. F. Ryba, and J. H. Taylor. This work was supported by the National Science Foundation (United States), via grants to Princeton University and support of the Arecibo Observatory (operated by Cornell University) and Green Bank and the VLA (operated by Associated Universities, Inc.).

\section{References}

Applegate, J. H. \& Shaham, J. 1994, ApJ, 436, 312

Arzoumanian, Z., Fruchter, A. S., \& Taylor, J. H. 1994, ApJ, 426, L85

Banit, M. \& Shaham, J. 1992, ApJ, 388, L19

Banit, M., Ruderman, M. A., Shaham, J., \& Applegate, J. H. 1993, ApJ, 415, 779

Camilo, F., Lorimer, D. R., Freire, P., Lyne, A. G., \& Manchester, R. N. 2000 ApJ, submitted

Fruchter, A. S., Stinebring, D. R. \& Taylor, J. H. 1988, Nature, 333, 237

Kaspi, V. M., Taylor, J. H., and Ryba, M. F. 1994, ApJ, 428, 713

Lyne, A. G., Biggs, J. D., Harrison, P. A., \& Bailes, M. 1993, Nature, 361, 47

Lyne, A. G., Johnston, S., Manchester, R. N., Staveley-Smith, L., \& D'Amico, N. 1990 , Nature, 347,650

Makishima, K., et al. 1981, ApJ, 247, L23

Nice, D. J. \& Thorsett, S. E. 1992, ApJ, 397, 249

Stappers et al. 1996, ApJ, 465, L119

Stappers, B. W., Bailes, M., Manchester, R. N., Sandhu, J. S., \& Toscano, M. 1998, ApJ, 499, 183 